\title{
Light-regulated Electrochemical Reaction: Alternative Strategy for Designing High-performance Gas Sensors
}

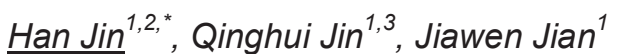 \\ ${ }^{1}$ School of Information Sciences and Technology, Ningbo University, Ningbo City, P. R. China, \\ ${ }^{2}$ Ningbo Institute of Industrial Technology, Ningbo City, P. R. China, \\ ${ }^{3}$ Shanghai Institute of Microsystem and Information Technology, Shanghai, P. R. China
}

Corresponding author's e-mail address: jinhan@nbu.edu.cn

\begin{abstract}
IMCS2018_Headline"):
Electrochemical gas sensors have been widely used for various applications. However, inadequate sensitive, detection limit and discrimination capability are still concerned issues for most of this type sensing device and restrains their research foreground. Beyond exploring well-designed sensing materials, newly reported light-regulated electrochemical reaction is considered as efficient sensing methodology to enforce sensing behavior of the gas sensors that operated with the lightregulated electrochemical sensing principle. In this article, a general summary is given to introduce relevant representative achievements made by Environmental Monitoring \& Sensing Technology Lab.(Ningbo University, P.R. China) during the past few years, whilst, strategy of designing highly sensitive \& selective solid-state electrochemical gas sensors will be particularly emphasized.
\end{abstract}

Key words: light-regulated electrochemical reaction, volatile organic compounds (VOCs), UV illumination, principle component analysis (PCA), discriminating features.

\section{Introduction}

Electrochemical gas sensor has been greatly regarded as one of the success representations among various solid-state gas sensing device [1]. However, inadequate sensitive, detection limit and discrimination feature are still remained challenging issues for electrochemical gas sensors and restrained their research foreground $[1,2]$. During the past decades, tons of relevant publications have been announced to eliminate the barrier. Although most of the work focus on exploring well-designed sensing materials, the rapid emergence of new materials with interesting properties significantly improved the practicability of applying this type of gas sensor in real use of various applications. Beyond continuously searching advanced sensing materials, quite recently light-regulated electrochemical reaction has been proposed and been confirmed to be effective approach for enhancing sensing behavior of electrochemical gas sensors. Herein, a general summary is given to introduce relevant representative achievements in improving the sensitivity and detection limit, meanwhile, helpful strategy to enforce the discriminating capability (or selectivity) of this type of sensor will be particularly emphasized.

\section{Experimental details}

Details of sensor fabrication can be found in previous report [2] and sensing performance of the sensors will be compared with (light on) or without (light off) UV illumination.

\section{Results and discussion}

\section{i. Light-regulated electrochemical reaction for aromatic VOCs detection}

UV illumination demonstrates obvious effects on the catalytic activity of photoactive SE, even when operated at high temperatures. The effect of UV light was strongly dependent on calcination temperature. With UV light, reactivity of the electrochemical reaction for both anodic and cathodic reaction appeared raised. Furthermore, the operating temperature of the sensor decreased without affecting its high performance. Based on this principle, sensing characteristic of the YSZ-based sensor (using ZnO-SE) towards several aromatic VOCs in the range of 4-14 ppm was examined (see Fig. 1). Significant enhancement was achieved in the sensitivity and detection limit, without affecting its intrinsic selectivity; notably the detection limit of the sensor to p-xylene increases from ppm to ppb level after exposure to UV light. Humidity had a negligible effect on the sensing performance of the sensor. 

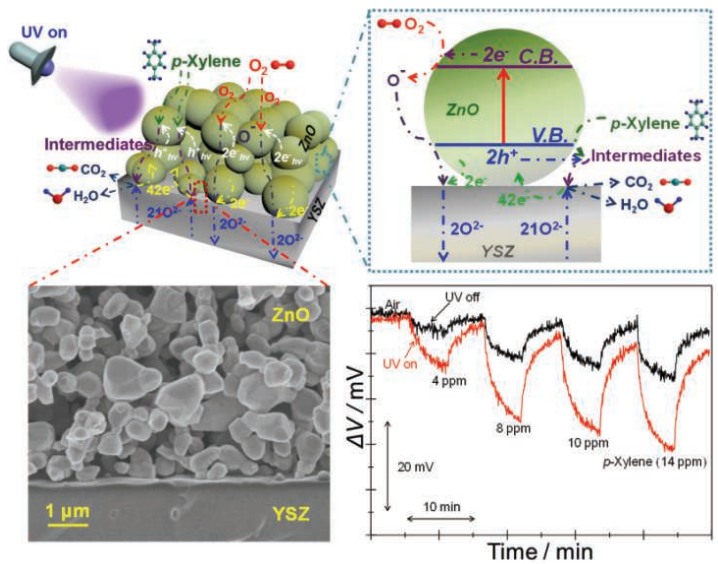

Fig. 1. Schema of the light regulated electrochemical reaction in enhancing the sensitivity and detection limit of zirconia-based gas sensor.

\section{ii. Further enhancement of the light- regulated potentiometric signal with composite electrode}

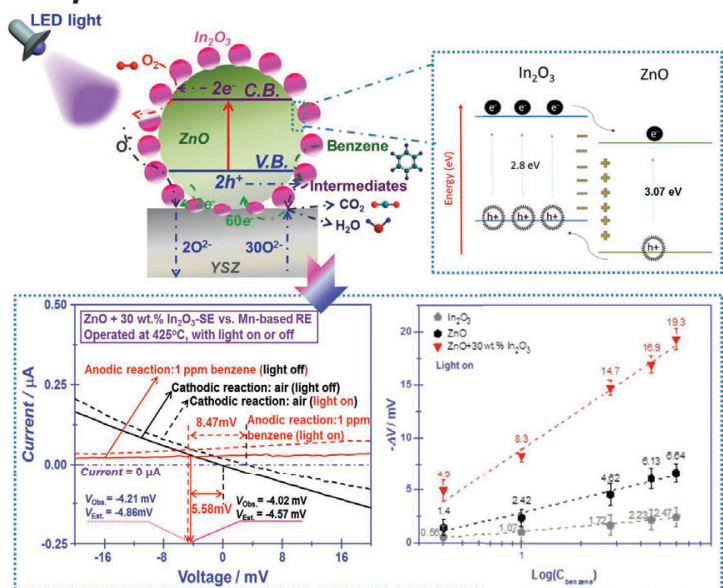

Fig. 2. Schema of further enhancement of mixpotential signal with ZnO-based electrode.

UV illumination has been confirmed to be essentially increased the response magnitude of potentiometric gas sensors. Nevertheless, the detection limit of the sensor is still inadequate for real application even upon UV light. In this section, the strategy of using more photoactive $\mathrm{ZnO}$-based composite as the SE for potentially further enhancing the sensing value is proposed and confirmed (see Fig.2). Typically, in comparison with ZnO-SE, ZnObased composite SE is beneficial for further promoting the light-regulation effect. Particularly, when upon illumination, the detection limit of the sensor consisting of the composite electrode (towards benzene) reaches $0.109 \mathrm{ppm}$, while that of the value for the sensor using $\mathrm{ZnO}-\mathrm{SE}$ is $0.898 \mathrm{ppm}$. The promoting mechanism is clarified from the perspective of the electrocatalytic activity \& gas-phase reactivity. In summary, forming the ZnO-based composites makes them ideal for generating abundant $\mathrm{e}-$ and $\mathrm{h}+$ when upon illumination, leading to the obvious increase in the catalytic activity of both cathodic \& anodic reaction and thereby further raised the mixedpotential signal.

\section{iii. Light-regulated Electrochemical Sensor Array for Discriminating Hazardous Gases}

A light-regulated electrochemical sensor array which comprised of 3 photosensitive sensor parts is fabricated and impact of illumination on the discrimination features of the sensor array is thoroughly studied (see Fig. 3). Typically, sensing response of the sensor array (upon illumination) is selectively enhanced to specific gases, leading to generating disparate response patterns when compared that of the sensor array operated without illumination. Hence, after processing all the response patterns (derived from light off \& on) with PCA algorithm, the discrimination capability for the sensor array is further improved. The impact mechanism of illumination is studied, it is deduced that effect of the illumination on the discriminating features can be attribute to the competition of photocatalytic activity \& gasphase reactivity.

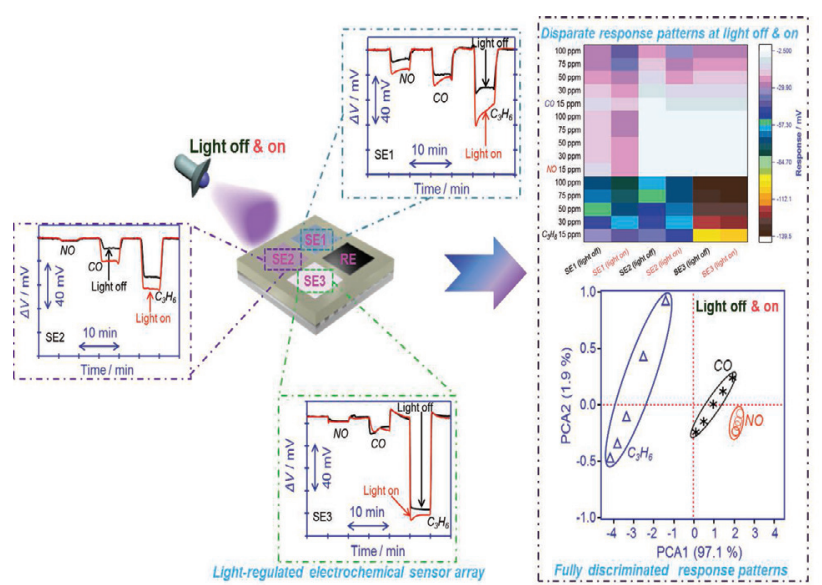

Fig. 3. Shema of the light-regulated electrochemical reaction for enforcing the discriminating features.

\section{Conclusions}

Light-regulated electrochemical reaction indeed essentially improved the performance of electrochemical gas sensors, we anticipate that the light-regulated electrochemical reaction will be a starting point for future designing of smarter sensing devices, opening a brighter future for progressively smarter monitoring of air quality \& human body conditions such as required in clinic diagnosis.

\section{References}

[1] N. Miura, T. Sato, S. A. Anggraini, H. Ikeda, S. Zhuiykov, A Review of Mixed-potential Type Zirconia-based Gas Sensors, lonics, 20, 901-25 (2014).; doi: 10.1007/s11581-014-1140-1

[2] H. Jin, H. Hossam, UV Regulation of Nonequilibrated Electrochemical Reaction for Detecting Aromatic Volatile Organic Compounds, Sensors and actuators B, 237, 30-40 (2016); doi: 10.1016/j.snb.2016.05.135 